\title{
SOME ANALYTICAL RESULTS FOR ACOUSTIC SCATTERING THROUGH A PERIODIC ARRAY OF ELASTIC MEMBRANES
}

\author{
$\mathrm{BY}$ \\ EDOARDO SCARPETTA (D.I.I.M.A., University of Salerno, 84084 Fisciano (SA), Italy) \\ AND \\ MEZHLUM A. SUMBATYAN (Faculty of Mechanics and Mathematics, Rostov State University, \\ 344090 Rostov-on-Don, Russia)
}

\begin{abstract}
In the context of wave propagation through acoustic media, an analytical approach is developed to study the (normal) incidence of a pressure wave into a periodic array of (thin) elastic membranes. The frequency of this wave is assumed in a range implying the so-called one-mode (far field) propagation, so that mild approximations holding in this range can be employed. Thus, the problem is reduced to some integral equations based on the opening between adjacent membranes and independent of the frequency. By means of the (analytical) solutions of such equations, an explicit formula for the transmission coefficient is set up and reflected in some figures for concrete values of the various parameters involved. The peculiarities of the scattering structure are discussed.
\end{abstract}

1. Introduction. The problems of solid-fluid interaction are very important in aeroacoustics, dynamics of aircrafts, architectural acoustics, active and passive noise control, as well as in many other fields of engineering science concerning vibrations of flexible constructions in contact with fluids and gases.

In this ambit, analytical methods certainly deserve great attention, since only they can provide explicit representations for the relevant unknown fields with respect to the geometrical or physical parameters involved.

The foundations of the dynamics of elastic constructions, in their interactions with fluids or gases, were posed by scientists in the theory of hydro-aero-elasticity (for a survey see, e.g., [1]-[3]). Several qualitatively interesting phenomena were discovered and studied, both in stationary and transient cases. Mathematically, the problems of such a kind are described by some system of integro-differential equations. Transient problems require an application of the Laplace transform with respect to time.

Practical applications of sound-elastic interaction are involved in noise control and sound insulation of buildings (see [4], Chapter 33). The theoretical basis for investigation

Received August 1, 2006.

2000 Mathematics Subject Classification. Primary 74K15.

(C)2007 Brown University 
of these matters, including the theory of sound transmission through thin elastic slabs, plates and membranes, can be found in [5]-[7]. A survey of simple analytical solutions and some aspects of direct numerical treatment by FEM, as well as a comparison with experiments, are presented in [8].

Noise control problems similar to those described above arise also in aeroacoustics in the ambit of reduction of noise generated by sound fields (see, for example, [9], Chapter 14). With this aim, some authors propose to apply linear arrays of membrane liners. Even simple impedance estimates show a wide range for efficient noise cancellation. More advanced theoretical investigations discover the so-called frequency cuts for the transmitted acoustic energy. This gives an alternative way to obtain noise control and sound insulation, along with an application of traditional absorbing materials. Mathematically, the "frequency cut" is related to a sudden decreasing of certain transmission coefficients when sound propagates through an acoustic medium containing some obstacles. Sound absorbers in the form of periodic collinear structures in room acoustics are called resonant absorbers. A special chapter is devoted to such absorbers in the leading book of Cremer and Müller [10], Chapter IV.9. The authors give many frequency-dependent graphs, basing their method on some simple approximate theories. They point out the relation of this approach with the theory of Helmholtz resonators.

An advanced analytical method for scattering problems through periodic structures of (thin) screens has been developed in our previous papersdouble [11, 12], where we were able to arrive at explicit representations of the scattered field, including transmission and reflection coefficients, in the case of absolutely rigid screens. In this connection, the main purpose of the present paper is to spread our method to the case of thin elastic screens (namely, membranes), in order to evaluate how significant can be the influence on the scattering of the flexibility of such (non-rigid) obstacles. The choice to consider the vibrating structure which interacts with the acoustic medium as (a periodic array of) elastic membranes is of course a first step towards the modelling of the physical problem here considered. In fact, some authors consider both the membrane and plate models when describing the fluid-elastic structures interaction; see, for example, [13]-[16] and the references therein cited. However, we should note that the modelling through membranes permits direct evaluation of the sound attenuation obtained by using soft elements (like curtains) in room acoustics. Indeed, it is well known [10] that to cancel too high reverberations in some types of halls (like sports halls or churches), the acousticians place soft thin materials in suitable parts of the hall. Thus, the present study gives a good mathematical instrument to calculate the level of transmission (and reflection) for such artificially arranged structures.

Through mild approximations involving the range of frequency under consideration, the scattering problem is reduced to certain integral equations (independent of frequency) originating by continuity assumptions and based on the opening between adjacent membranes; such equations are then analytically solved and some related integrals calculated, so as to provide an explicit formula for the transmission coefficient with respect to frequency. The results are reflected in several figures for concrete values of the geometrical and physical parameters. The wave properties of the scattering structure are finally discussed. In an Appendix the main technical transformations are reported. 
2. Formulation of the problem and reduction to integral equations. We consider an unbounded acoustic medium of density $\rho_{a}$ in which there is a periodic distribution of coplanar parallel (thin and flat) elastic membranes, each one subjected to the same (given) biaxial tension. If $z$ denotes the axis along which such (infinite) membranes run, Figure 1 shows the section of the structure with (any) normal plane $x y$. The period of the array is $2 a$, while the width of each membrane is $2 b(b<a)$.

In the harmonic regime, we assume that a plane pressure wave of given amplitude $p_{0}$ and circular frequency $\omega$,

$$
p_{\text {inc }}=p_{o} e^{i(k x-\omega t)},
$$

is incident from $x=-\infty$ onto the membranes' array, giving rise to scattered waves on the left $(x<0)$ and right $(x>0)$ sides of the structure. Above, $k$ is the wave number, and $\omega / k$ gives the (constant) wave speed in the medium. Throughout the sequel, the time dependence factor $e^{-i \omega t}$ is common and will often be omitted.

Thanks to the natural symmetry and periodicity, the problem can be restricted to the typical strip $|y|<a$, in which the (section of a) membrane occupies the segment $|y|<b$ at $x=0$. By the same reason, the following Fourier representations can be given for the total field in the two main regions of the structure:

$$
\begin{array}{cc}
p_{\text {left }}(x, y)=p_{0} e^{i k x}+p_{0} R e^{-i k x}+\sum_{n=1}^{+\infty} A_{n} e^{q_{n} x} \cos \frac{\pi n}{a} y, & x<0, \\
p_{\text {right }}(x, y)=p_{0} T e^{i k x}+\sum_{n=1}^{+\infty} B_{n} e^{-q_{n} x} \cos \frac{\pi n}{a} y, & x>0,
\end{array}
$$

where all capital letters denote unknown constants and

$$
q_{n}=\sqrt{(\pi n / a)^{2}-k^{2}}, \quad n=1,2, \ldots
$$

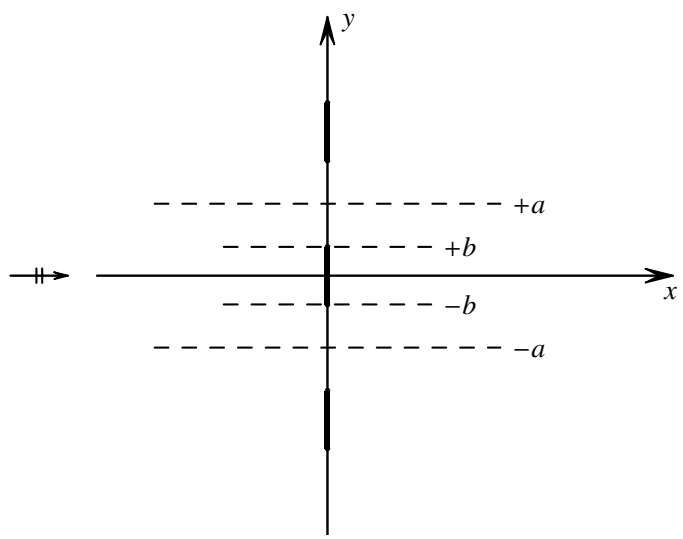

FIG. 1. Normal incidence of a pressure wave into a periodic array of elastic membranes (of width $2 b$ ) in an acoustic medium. The period is $2 a$. 
in order that each wave mode in the series above be a trivial solution of the governing (Helmholtz) equation

$$
\frac{\partial^{2} p}{\partial x^{2}}+\frac{\partial^{2} p}{\partial y^{2}}+k^{2} p=0
$$

In such a series, we prefer to extract the terms with $n=0$, putting $A_{0}=p_{0} R, B_{0}=p_{0} T$; $q_{0}=-i k$ by a radiation condition. As it is due, $\partial p / \partial y=0 \forall x$ at $y= \pm a$.

Taking $\omega$ in the range such that

$$
(0<) k<\pi / a
$$

implies $q_{n}>0 \forall n \geq 1$, and thus guarantees the so-called one-mode (far field) propagation, since with distance from the membranes only the wave modes of order zero will remain non-vanishing in Eqs.(2.2). In this case, the corresponding (non-dimensional) coefficients $R$ and $T$ are referred to as the scattering parameters of the structure.

The (linear) motion of an elastic membrane surrounded by a pressure field $p$ is governed by the following equation $[7,8]$ :

$$
\tau \nabla^{2} \hat{w}=\rho_{e} \frac{\partial^{2} \hat{w}}{\partial t^{2}}+p
$$

where $\hat{w}$ is the (out-of-plane) displacement field, while $\tau$ and $\rho_{e}$ are positive constants which denote the given biaxial tension (force per unit length, here along $z$ ) and material density (mass per unit area) of the membrane, respectively. Of course, in the present (linear) context the pressure wave field interacts with the membranes imposing a vibrating motion of harmonic type with the same circular frequency $\omega[7,8]$. As a consequence, the stationary governing equation for the $x$-component (only non-trivial) of the displacement field of the typical membrane, $\hat{w}(y, t)=e^{-i \omega t} w(y)$, reads now as

$$
\tau \frac{d^{2}}{d y^{2}} w(y)+\rho_{e} \omega^{2} w(y)=p_{\text {right }}(0, y)-p_{\text {left }}(0, y), \quad|y|<b .
$$

By symmetry, $w(y)$ is an even function.

The membrane is clamped at $|y|=b$, so that as a boundary condition we have

$$
w( \pm b)=0 \text {. }
$$

Moreover, the pressure field on the membrane should be coupled with the motion of the membrane by equating the $x$-components of the velocities at $x=0$ :

$$
\frac{\partial}{\partial t} \hat{w}(y, t)=v_{a}(y, t), \quad|y|<b
$$

where that component of the velocity field in the acoustic medium, $v_{a}$, is here governed by the equation

$$
\rho_{a} \frac{\partial v_{a}}{\partial t}=-\left.e^{-i \omega t} \frac{\partial p}{\partial x}\right|_{x=0}
$$

Such a coupling clearly implies

$$
\left.\frac{\partial p_{\text {left }}}{\partial x}\right|_{x=0}=\left.\frac{\partial p_{\text {right }}}{\partial x}\right|_{x=0}, \quad|y|<b,
$$


which, along with an obvious property of continuity for the above derivatives on $b<$ $|y|<a$, finally enables us to put

$$
\left.\frac{\partial p_{\text {left }}}{\partial x}\right|_{x=0}=\left.\frac{\partial p_{\text {right }}}{\partial x}\right|_{x=0}, \quad|y|<a .
$$

Recalling Eqs.(2.2), Eq.(2.10) and the orthogonality properties of cosines in $|y|<a$ directly implies

$$
1-R=T, \quad B_{n}=-A_{n} .
$$

Now, let us go back to Eq.(2.6a), and substitute $p_{\text {right }}(0, y)-p_{\text {left }}(0, y)$ from Eqs.(2.2) taking into account Eqs.(2.11); we get

$$
\frac{d^{2}}{d y^{2}} w(y)+\frac{\rho_{e} \omega^{2}}{\tau} w(y)=\left(2 p_{0} / \tau\right)(T-1)+(2 / \tau) \sum_{n=1}^{+\infty} B_{n} \cos \frac{\pi n}{a} y, \quad|y|<b,
$$

which implies the (even) solution

$$
w(y)=c_{1} \cos \Omega y-\frac{2}{\tau} \sum_{n=1}^{+\infty} \frac{B_{n}}{s_{n}^{2}} \cos \frac{\pi n}{a} y+\frac{2 p_{0}}{\rho_{e} \omega^{2}}(T-1), \quad|y|<b,
$$

where we put

$$
\Omega=\sqrt{\rho_{e} \omega^{2} / \tau}, \quad s_{n}=\sqrt{(\pi n / a)^{2}-\Omega^{2}} .
$$

By using Eq.(2.6b), we obtain the following explicit expression for the displacement field of the membrane:

$$
\begin{gathered}
w(y)=\frac{2 p_{0}}{\rho_{e} \omega^{2}}(T-1)+\left[\frac{2 p_{0}}{\rho_{e} \omega^{2}}(1-T)+\frac{2}{\tau} \sum_{n=1}^{+\infty} \frac{B_{n}}{s_{n}^{2}} \cos \frac{\pi n}{a} b\right] \frac{\cos \Omega y}{\cos \Omega b} \\
-\frac{2}{\tau} \sum_{n=1}^{+\infty} \frac{B_{n}}{s_{n}^{2}} \cos \frac{\pi n}{a} y, \quad|y|<b .
\end{gathered}
$$

In the assumed harmonic regime, substituting Eq.(2.7) into Eq.(2.8) and considering Eq.(2.10) give

$$
w(y)=\frac{1}{\rho_{a} \omega^{2}} \frac{\partial p_{\text {right }}}{\partial x}(0, y), \quad|y|<b .
$$

Now, the idea is to define a new unknown function $g(y)$ on the opening $b<|y|<a$, as follows:

$$
w(y)-\frac{1}{\rho_{a} \omega^{2}} \frac{\partial p_{\text {right }}}{\partial x}(0, y)=\left\{\begin{array}{l}
g(y), \quad b<|y|<a \\
0, \quad|y|<b
\end{array}\right.
$$

where by $w(y)$ on the opening we mean the function on the right-hand side of Eq.(2.14) accordingly extended. Clearly, $g(y)$ is even.

Let us put

$$
G(n)=\int_{b}^{a} g(t) \cos \frac{\pi n}{a} t d t, \quad n=0,1,2, \ldots ; \quad G=G(0)\left(=\int_{b}^{a} g(t) d t\right) .
$$


By using Eq.(2.2b), we can integrate Eq.(2.16) over the total width $|y|<a$ to obtain the following formula for the coefficient $T$ (in terms of $B_{n}$ ) :

$$
\begin{gathered}
p_{0}\left[\frac{1}{\rho_{e}}\left(1-\frac{\sin \Omega a}{\Omega a \cos \Omega b}\right)-\frac{i k}{2 \rho_{a}}\right] T=\frac{\omega^{2}}{2 a}\left[G+\frac{2 a p_{0}}{\rho_{e} \omega^{2}}\left(1-\frac{\sin \Omega a}{\Omega a \cos \Omega b}\right)\right. \\
\left.-\frac{2 a}{\tau} \frac{\sin \Omega a}{\Omega a \cos \Omega b} \sum_{n=1}^{+\infty} \frac{B_{n}}{s_{n}^{2}} \cos \frac{\pi n}{a} b\right] .
\end{gathered}
$$

Repeating the integration of Eq.(2.16) after multiplying by $\cos (\pi m y / a), m=1,2, \ldots$, gives

$$
\begin{gathered}
\left(\frac{a q_{m}}{2 \rho_{a} \omega^{2}}-\frac{a}{\tau s_{m}^{2}}\right) B_{m}=G(m)+\frac{2 \Omega}{\rho_{e} \omega^{2}} \frac{\sin \Omega a}{\cos \Omega b} \frac{(-1)^{m}}{s_{m}^{2}} p_{0}(1-T) \\
+\frac{2 \Omega}{\tau} \frac{\sin \Omega a}{\cos \Omega b} \frac{(-1)^{m}}{s_{m}^{2}} \sum_{n=1}^{+\infty} \frac{B_{n}}{s_{n}^{2}} \cos \frac{\pi n}{a} b, \quad m=1,2, \ldots
\end{gathered}
$$

The series above can be substituted from Eq. (2.18), so that we can also deduce a formula for the coefficients $B_{n}$ (in terms of $T$ ), as follows:

$$
\begin{gathered}
\left(\frac{a q_{n}}{2 \rho_{a} \omega^{2}}-\frac{a}{\tau s_{n}^{2}}\right) B_{n}=G(n)+\frac{(-1)^{n}}{s_{n}^{2}}\left(\Omega^{2} G+\frac{2 a p_{0}}{\tau}\right) \\
-\frac{a}{\tau} \frac{(-1)^{n}}{s_{n}^{2}}\left(2-i k \frac{\rho_{e}}{\rho_{a}}\right) p_{0} T, \quad n=1,2, \ldots .
\end{gathered}
$$

Of course, inserting Eq.(2.20) into Eq.(2.18) would give a final formula containing only $T$ versus the integrals (2.17) of function $g$, which thus remains the main unknown of the problem.

3. Integral equation. Quasi-one-mode approximation. An integral equation to be satisfied by function $g$ arises from the assumption of continuity for the pressure field along the opening, as follows:

$$
p_{\text {right }}(0, y)=p_{\text {left }}(0, y), \quad b<|y|<a .
$$

By using Eqs.(2.2) and (2.11), we easily deduce

$$
\sum_{n=1}^{+\infty} B_{n} \cos \frac{\pi n}{a} y=p_{0}(1-T), \quad b<|y|<a,
$$

in which one could imagine substituting $B_{n}, T$ from system $(2.18,2.20)$. That would lead to an (exact) integral equation, which of course could be directly submitted to standard numerical algorithms for arbitrary values of the geometrical and physical parameters. However, this paper aims to remain in a full-analytical context, and to this end we agree to make the following approximation: recalling the range of frequency in (2.4), we assume to be negligible each term containing $(a k)^{2}$ when compared with $(\pi n)^{2} \quad \forall n=1,2, \ldots$; 
this implies that

$$
q_{n} \simeq \frac{\pi n}{a}, \quad s_{n} \simeq \frac{\pi n}{a}, \quad n=1,2, \ldots{ }^{1}
$$

(In some power expansions, the term $\Omega a$ will be retained up to and including the third power.)

As a consequence, formulas for $B_{n}, T$ become

$$
\begin{gathered}
B_{n} \simeq \frac{2 \rho_{a} \omega^{2}}{\pi}\left[\frac{G(n)}{n}+\frac{2 a^{3} p_{0}}{\tau} \frac{(-1)^{n}}{\pi^{2} n^{3}}-\frac{a^{3}}{\tau} \frac{(-1)^{n}}{\pi^{2} n^{3}}\left(2-i k \frac{\rho_{e}}{\rho_{a}}\right) p_{0} T\right], \\
\left(\frac{a^{3}-3 a b^{2}}{3 \tau}-\frac{a i k}{\rho_{a} \omega^{2}}\right) p_{0} T \simeq G+\frac{a^{3}-3 a b^{2}}{3 \tau} p_{0}-\frac{2 a^{3}}{\tau \pi^{2}} \frac{\sin \Omega a}{\Omega a \cos \Omega b} \sum_{n=1}^{+\infty} \frac{B_{n}}{n^{2}} \cos \frac{\pi n}{a} b .
\end{gathered}
$$

In Eq.(3.5) we can substitute $B_{n}$ from Eq.(3.4) to get

$$
\begin{aligned}
& {\left[A-\frac{a i k}{\rho_{a} \omega^{2}}-B C \rho_{a} \omega^{2} \frac{\sin \Omega a}{\Omega a \cos \Omega b}\left(2-i k \frac{\rho_{e}}{\rho_{a}}\right)\right] p_{0} T} \\
& \quad=G+A p_{0}-B \rho_{a} \omega^{2} \frac{\sin \Omega a}{\Omega a \cos \Omega b} \sum_{n=1}^{+\infty} \frac{G(n)}{n^{3}} \cos \frac{\pi n}{a} b-2 B C \rho_{a} \omega^{2} \frac{\sin \Omega a}{\Omega a \cos \Omega b} p_{0}
\end{aligned}
$$

where for convenience we have put

$$
A=\frac{a^{3}-3 a b^{2}}{3 \tau}, \quad B=\frac{4 a^{3}}{\tau \pi^{3}}, \quad C=\frac{a^{3}}{\tau \pi^{2}} \sum_{n=1}^{+\infty} \frac{(-1)^{n}}{n^{5}} \cos \frac{\pi n}{a} b,
$$

three structural constants which are free of frequency. The dependence on frequency in the above formula remains implicit in the terms involving (the integrals of) function $g$.

By substituting (only) $B_{n}$ from Eq.(3.4), Eq.(3.2) gives an integral equation for $g$ as follows:

$$
\begin{aligned}
\int_{b}^{a}\left(\sum_{n=1}^{+\infty} \frac{1}{n} \cos \frac{\pi n}{a} t \cos \frac{\pi n}{a} y\right) g(t) d t=\frac{\pi p_{0}}{2 \rho_{a} \omega^{2}}(1-T) \\
+\frac{a^{3} p_{0}}{\tau \pi^{2}}\left[\left(2-i k \frac{\rho_{e}}{\rho_{a}}\right) T-2\right] \sum_{m=1}^{+\infty} \frac{(-1)^{m}}{m^{3}} \cos \frac{\pi m}{a} y, \quad b<y<a .
\end{aligned}
$$

For technical reasons (connected to the solution of the forthcoming auxiliary integral equations), we prefer to base Eq.(3.7) over the whole opening $(-d,+d)$, where $d=a-b$. Thus, by shifting the $x$-axis up to $y=a$ and an even extension of the function $g$ from $(-d, 0)$ to $(-d,+d)$, Eq. $(3.7)$ becomes

$$
\begin{aligned}
& \frac{1}{2} \int_{-d}^{d}\left(\sum_{n=1}^{+\infty} \frac{1}{n} \cos \frac{\pi n(t-y)}{a}\right) g(t) d t=\frac{\pi p_{0}}{2 \rho_{a} \omega^{2}}(1-T) \\
& +\frac{a^{3} p_{0}}{\tau \pi^{2}}\left[\left(2-i k \frac{\rho_{e}}{\rho_{a}}\right) T-2\right] \sum_{m=1}^{+\infty} \frac{1}{m^{3}} \cos \frac{\pi m}{a} y, \quad|y|<d,
\end{aligned}
$$

\footnotetext{
${ }^{1}$ In the strict one-mode approximation, similar positions are made only for $n \geq 2[11,12]$; this justifies the terminology used in the section's title.
} 
keeping for simplicity the same symbols for $g, t, y$. Of course, after this transformation, in Eq.(3.6a) for $T$ it follows that

$$
G=\frac{1}{2} \int_{-d}^{d} g(t) d t, \quad G(n)=\frac{(-1)^{n}}{2} \int_{-d}^{d} g(t) \cos \frac{\pi n}{a} t d t
$$

Moreover, in the kernel above we can put $\sum_{n=1}^{+\infty} \frac{1}{n} \cos \frac{\pi n(t-y)}{a}=-\ln \left|2 \sin \frac{\pi(t-y)}{2 a}\right|$.

Now, it is clear that if one solves the following auxiliary integral equations,

$$
-\frac{1}{2} \int_{-d}^{d} \ln \left|2 \sin \frac{\pi(t-y)}{2 a}\right| g_{m}(t) d t=\cos \frac{\pi m y}{a}, \quad m=0,1,2, \ldots, \quad|y|<d \quad(d<a),
$$

each one free of any frequency parameter, then, by linearity, the solution of Eq.(3.8) can be constructed as

$$
g(t)=\frac{\pi p_{0}}{2 \rho_{a} \omega^{2}}(1-T) g_{0}(t)+\frac{a^{3} p_{0}}{\tau \pi^{2}}\left[\left(2-i k \frac{\rho_{e}}{\rho_{a}}\right) T-2\right] \sum_{m=1}^{+\infty} \frac{1}{m^{3}} g_{m}(t), \quad|t|<d .
$$

This gives for constants $G, G(n)$ in Eqs.(3.9) the expressions

$$
\begin{aligned}
G & =\frac{\pi p_{0}}{2 \rho_{a} \omega^{2}}(1-T) G_{0}^{0}+\frac{a^{3} p_{0}}{\tau \pi^{2}}\left[\left(2-i k \frac{\rho_{e}}{\rho_{a}}\right) T-2\right] \sum_{m=1}^{+\infty} \frac{1}{m^{3}} G_{m}^{0}, \\
G(n) & =\frac{\pi p_{0}}{2 \rho_{a} \omega^{2}}(1-T) G_{0}^{n}+\frac{a^{3} p_{0}}{\tau \pi^{2}}\left[\left(2-i k \frac{\rho_{e}}{\rho_{a}}\right) T-2\right] \sum_{m=1}^{+\infty} \frac{1}{m^{3}} G_{m}^{n}
\end{aligned}
$$

where we have introduced the constants

$$
G_{m}^{n}=\frac{(-1)^{n}}{2} \int_{-d}^{d} g_{m}(t) \cos \frac{\pi n}{a} t d t, \quad m, n=0,1,2, \ldots
$$

which are free of frequency. Going back to Eq.(3.6a) and substituting there $G, G(n)$ from (3.12), we finally have a formula for the transmission coefficient $T$ in which dependence on frequency appears in explicit form:

$$
\begin{gathered}
{\left[A-\frac{a i k}{\rho_{a} \omega^{2}}-B C \rho_{a} \omega^{2} \frac{\sin \Omega a}{\Omega a \cos \Omega b}\left(2-i k \frac{\rho_{e}}{\rho_{a}}\right)\right] T=A+\frac{\pi / 2}{\rho_{a} \omega^{2}}(1-T) G_{0}^{0}} \\
+\frac{a^{3}}{\tau \pi^{2}}\left[\left(2-i k \frac{\rho_{e}}{\rho_{a}}\right) T-2\right] \sum_{m=1}^{+\infty} \frac{1}{m^{3}} G_{m}^{0}-B \rho_{a} \omega^{2} \frac{\sin \Omega a}{\Omega a \cos \Omega b}\{2 C \\
\left.+\frac{\pi / 2}{\rho_{a} \omega^{2}}(1-T) \sum_{n=1}^{+\infty} \frac{1}{n^{3}} G_{0}^{n} \cos \frac{\pi n}{a} b+\frac{a^{3}}{\tau \pi^{2}}\left[\left(2-i k \frac{\rho_{e}}{\rho_{a}}\right) T-2\right] \sum_{m, n=1}^{+\infty} \frac{1}{m^{3} n^{3}} G_{m}^{n} \cos \frac{\pi n}{a} b\right\} .
\end{gathered}
$$


4. Analytical solution of auxiliary integral equations and calculation of constants $\mathbf{G}_{m}^{n}$. Let us firstly introduce the new functions

$$
\hat{\psi}_{m}(y)=\int_{-d}^{y} g_{m}(t) d t, \quad \hat{\psi}_{m}(-d)=0, \quad \hat{\psi}_{m}(d)=2 G_{m}^{0}, \quad m=0,1,2, \ldots,
$$

and apply integration by parts in Eq.(3.10); we get

$$
-\ln \left|2 \sin \frac{\pi(d-y)}{2 a}\right| G_{m}^{0}+\frac{\pi}{4 a} \int_{-d}^{d} \cot \frac{\pi(t-y)}{2 a} \hat{\psi}_{m}(t) d t=\cos \frac{\pi m y}{a}, \quad|y|<d .
$$

By the change of variables

$$
z=e^{\pi i y / a}, \quad \tau=e^{\pi i t / a} \quad(i \pi d t=a d \tau / \tau)
$$

it follows that

$$
\begin{aligned}
\ln \left|2 \sin \frac{\pi(d-y)}{2 a}\right| & =\ln \left(e^{i \pi d / a}-z\right)-\frac{i \pi}{2}\left(1+\frac{d}{a}\right)-\frac{1}{2} \ln z, \\
\cot \frac{\pi(y-t)}{2 a} & =i \frac{z+\tau}{z-\tau}
\end{aligned}
$$

so that Eq.(4.2) can be rewritten in complex form as

$$
\begin{gathered}
\frac{1}{i \pi} \int_{\alpha}^{\beta} \frac{\psi_{m}(\tau)}{\tau-z} d \tau=F_{m}(z), \quad z \in L(\alpha, \beta), \quad m=0,1,2, \ldots, \\
F_{m}(z) \equiv \frac{\Psi_{m}}{2 a}+\frac{2 G_{m}^{0}}{i \pi}\left[\ln (\beta-z)-\frac{i \pi}{2}\left(1+\frac{d}{a}\right)-\frac{1}{2} \ln z\right]+\frac{1}{i \pi}\left(z^{m}+z^{-m}\right),
\end{gathered}
$$

where $\psi_{m}(\tau$ or $z)=\hat{\psi}_{m}(t$ or $y), \Psi_{m} \equiv \int_{-d}^{d} \hat{\psi}_{m}(t) d t=\frac{a}{i \pi} \int_{\alpha}^{\beta} \psi_{m}(\tau) d \tau / \tau$, and the contour $L(\alpha, \beta)=\left\{z=e^{i \phi},|\phi|<\pi d / a\right\}$ is an open part of the unit circle in the complex plane; $\alpha=e^{-i \pi d / a}, \beta=e^{i \pi d / a}=\alpha^{-1}$, and the symbol $\int_{\alpha}^{\beta}(\ldots)$ means throughout integration over such a contour.

According to the classical theory of singular integral equations of Cauchy type [17], the general solution of Eq.(4.5) can be given as

$$
\psi_{m}(z)=\frac{1}{\pi i \sqrt{(z-\alpha)(\beta-z)}}\left[C+\int_{\alpha}^{\beta} \frac{F_{m}(\xi) \sqrt{(\xi-\alpha)(\beta-\xi)}}{\xi-z} d \xi\right], \quad z \in L(\alpha, \beta),
$$

where $C$ is an arbitrary constant while $\Psi_{m}, G_{m}^{0}$ appear as unknown constants inside $F_{m}(\cdot)$.

By requiring boundedness of the solution (4.6) as $z \rightarrow \alpha$, we get a particular value for the constant $C$,

$$
C=-\int_{\alpha}^{\beta} F_{m}(\xi) \sqrt{\frac{\beta-\xi}{\xi-\alpha}} d \xi
$$


so that Eq.(4.6) itself becomes:

$$
\psi_{m}(z)=\frac{1}{\pi i} \sqrt{\frac{z-\alpha}{\beta-z}} \int_{\alpha}^{\beta} F_{m}(\xi) \sqrt{\frac{\beta-\xi}{\xi-\alpha}} \frac{d \xi}{\xi-z}, \quad z \in L(\alpha, \beta) .
$$

Imposing also boundedness of (4.8) as $z \rightarrow \beta$ gives the following condition:

$$
\int_{\alpha}^{\beta} \frac{F_{m}(\xi)}{\sqrt{(\xi-\alpha)(\beta-\xi)}} d \xi=0
$$

which actually implies a linear relation between the two unknowns $\Psi_{m}, G_{m}^{0}$. Another linear relation can be obtained by dividing Eq.(4.8) by $z$ and integrating over $L(\alpha, \beta)$; the result is

$$
\frac{\pi}{a} \Psi_{m}=\int_{\alpha}^{\beta} \frac{F_{m}(\xi) d \xi}{\xi \sqrt{(\xi-\alpha)(\beta-\xi)}}
$$

where integral (A.5) has been used (see Appendix) and Eq.(4.9) taken into account.

It is clear that, after substituting $F_{m}(\cdot)$ from Eq.(4.5b), Eqs.(4.9) and (4.10) represent a $2 \times 2$ linear algebraic system for determining $\Psi_{m}, G_{m}^{0}$. To render it explicit implies evaluation of several integrals, as shown in the Appendix; see integrals (A.b, A.1, A.2, A.6, A.7, A.8, A.9).

One finally gets

$$
\left\{\begin{array}{c}
\frac{\pi}{2 a} \Psi_{m}+\frac{2 G_{m}^{0}}{i}\left[\ln \left(\sin \frac{\pi d}{2 a}\right)-\frac{\pi i d}{2 a}\right]+\frac{J_{m}+J_{m-1}}{\pi i}=0 \\
\frac{\pi}{2 a} \Psi_{m}-\frac{2 G_{m}^{0}}{i}\left[\ln \left(\sin \frac{\pi d}{2 a}\right)+\frac{\pi i d}{2 a}\right]-\frac{J_{m}+J_{m-1}}{\pi i}=0
\end{array}\right.
$$

whose solution is easily given by

$$
G_{m}^{0}=-\frac{J_{m}+J_{m-1}}{2 \pi \ln \left(\sin \frac{\pi d}{2 a}\right)}, \quad \Psi_{m}=2 d G_{m}^{0}=-\frac{d\left(J_{m}+J_{m-1}\right)}{\pi \ln \left(\sin \frac{\pi d}{2 a}\right)} .
$$

By integration by parts and change of variables (4.3), the main constants $G_{m}^{n}$ in Eqs.(3.12c) and (3.13), can be expressed as follows:

$$
\begin{aligned}
& (-1)^{n} G_{m}^{n}=G_{m}^{0} \cos \frac{\pi n d}{a}+\frac{\pi n}{2 a} \int_{-d}^{d} \hat{\psi}_{m}(t) \sin \frac{\pi n t}{a} d t \\
& =G_{m}^{0} \cos \frac{\pi n d}{a}-\frac{n}{4} \int_{\alpha}^{\beta} \psi_{m}(\tau)\left(\tau^{n-1}-\tau^{-n-1}\right) d \tau .
\end{aligned}
$$

On using Eqs.(4.8) and (4.9), it is not difficult to get

$$
\int_{\alpha}^{\beta} \psi_{m}(\tau)\left(\begin{array}{c}
\tau^{n-1} \\
\tau^{-n-1}
\end{array}\right) d \tau=\frac{1}{i \pi} \int_{\alpha}^{\beta} \frac{F_{m}(\xi)}{\sqrt{(\xi-\alpha)(\beta-\xi)}}\left(\begin{array}{c}
N_{n-1}(\xi) \\
N_{-n-1}(\xi)
\end{array}\right) d \xi \quad(n=1,2, \ldots)
$$


where the integrals

$$
\begin{gathered}
N_{n-1}(\xi) \equiv \int_{\alpha}^{\beta} \frac{\sqrt{(\tau-\alpha)(\beta-\tau)}}{\xi-\tau} \tau^{n-1} d \tau \\
=-\left(\sum_{j=0}^{n-2}\right)^{\prime} \xi^{n-2-j} I_{j}+\pi \xi^{n}-\pi \xi^{n-1} \cos \frac{\pi d}{a} \\
N_{-n-1}(\xi) \equiv \int_{\alpha}^{\beta} \frac{\sqrt{(\tau-\alpha)(\beta-\tau)}}{\xi-\tau} \tau^{-n-1} d \tau=-(1 / \xi) N_{n-1}(1 / \xi), \quad n=1,2, \ldots,
\end{gathered}
$$

are calculated in the Appendix (see (A.10, A.11)); the braces with a prime around the summation above mean that such a term is absent when $n=1$ (also in the forthcoming Eq.(4.16)).

Equation (4.13), by using Eqs.(4.14,4.15) and substituting $F_{m}(\cdot)$ from $(4.5 \mathrm{~b})$, leads to

$$
\begin{gathered}
(-1)^{n} G_{m}^{n}=G_{m}^{0} \cos \frac{\pi n d}{a}-\frac{n}{4 \pi i} \int_{\alpha}^{\beta} \frac{F_{m}(\xi)\left[N_{n-1}(\xi)-N_{-n-1}(\xi)\right]}{\sqrt{(\xi-\alpha)(\beta-\xi)}} d \xi \\
=G_{m}^{0} \cos \frac{\pi n d}{a}+\frac{n}{4 \pi i} \int_{\alpha}^{\beta}\left\{\frac{\Psi_{m}}{2 a}+\frac{2 G_{m}^{0}}{i \pi}\left[\ln (\beta-\xi)-\frac{i \pi}{2}\left(1+\frac{d}{a}\right)-\frac{1}{2} \ln \xi\right]\right. \\
\times\left[\left(\sum_{j=0}^{n-2}\right)^{\prime}\left(\xi^{n-j-2}+\xi^{j-n+1}\right) I_{j}-\pi\left(\xi^{n}+\xi^{-n-1}\right)\right. \\
\left.+\pi\left(\xi^{n-1}+\xi^{-n}\right) \cos \frac{\pi d}{a}\right] \frac{d \xi}{\sqrt{(\xi-\alpha)(\beta-\xi)}}
\end{gathered}
$$

Besides integrals of type $J_{ \pm k}$ (see (A.b, A.1) of Appendix), the new integrals with logarithmic integrand which now appear,

$$
P_{ \pm k}=\int_{\alpha}^{\beta} \xi^{ \pm k} \frac{\ln (\beta-\xi)}{\sqrt{(\xi-\alpha)(\beta-\xi)}} d \xi, \quad Q_{ \pm k}=\int_{\alpha}^{\beta} \xi^{ \pm k} \frac{\ln \xi}{\sqrt{(\xi-\alpha)(\beta-\xi)}} d \xi
$$

are calculated in the Appendix; see (A.12, A.18, A.20, A.21).

As a result of all the previous calculations, the (analytic) expression of $G_{m}^{n}$ can be used in formula (3.13). 


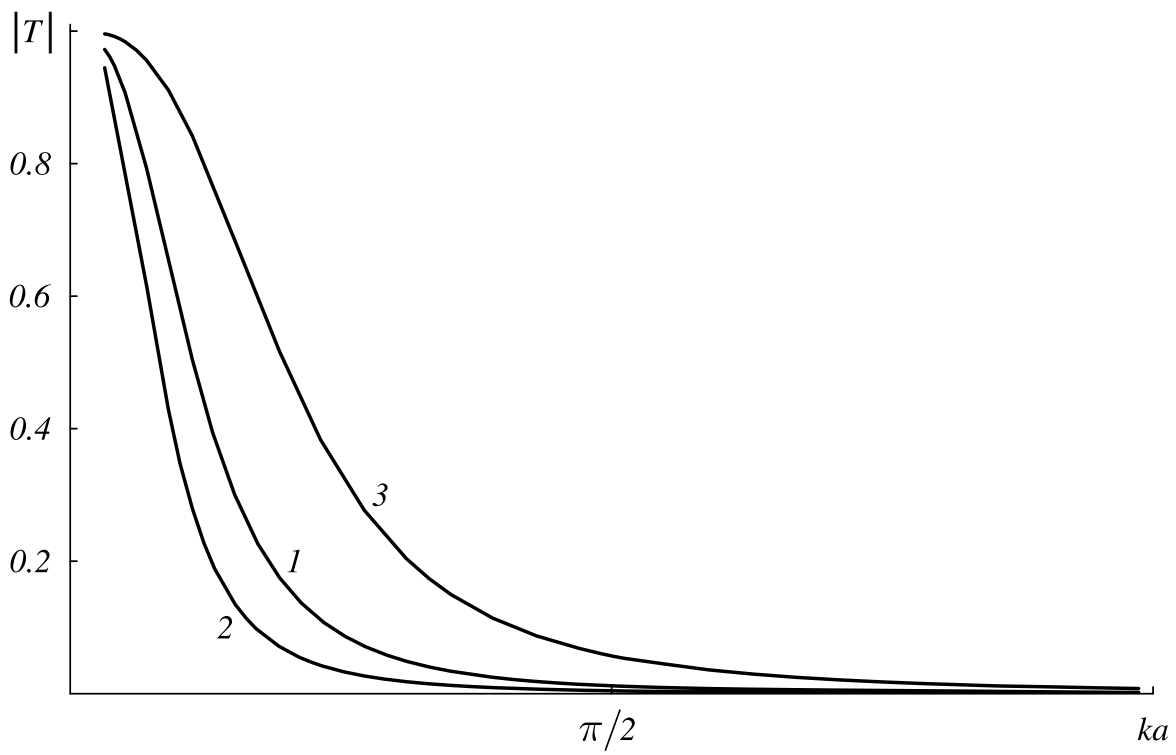

FIG. 2. Transmission coefficient vs. frequency parameter $a k<\pi$, for (relatively) very small tension of membranes: $\hat{\tau} \equiv \tau /\left(\rho_{e} c^{2}\right)=10^{-4}$. Line $1: b / a=0.1$; line $2: b / a=0.5$; line $3: b / a=0.9$.

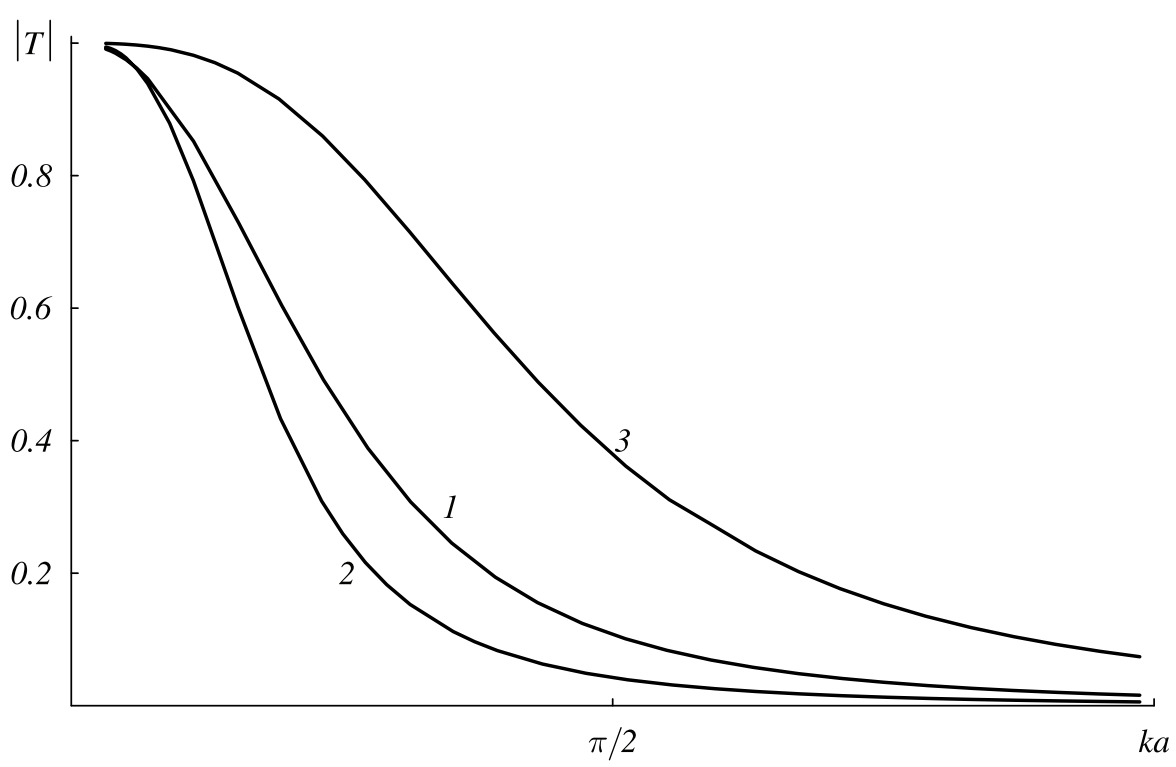

FIG. 3. Transmission coefficient vs. frequency parameter $a k<\pi$, for (relatively) small tension of membranes: $\hat{\tau} \equiv \tau /\left(\rho_{e} c^{2}\right)=10^{-3}$. Line $1: b / a=0.1 ;$ line $2: b / a=0.5 ;$ line $3: b / a=0.9$ 


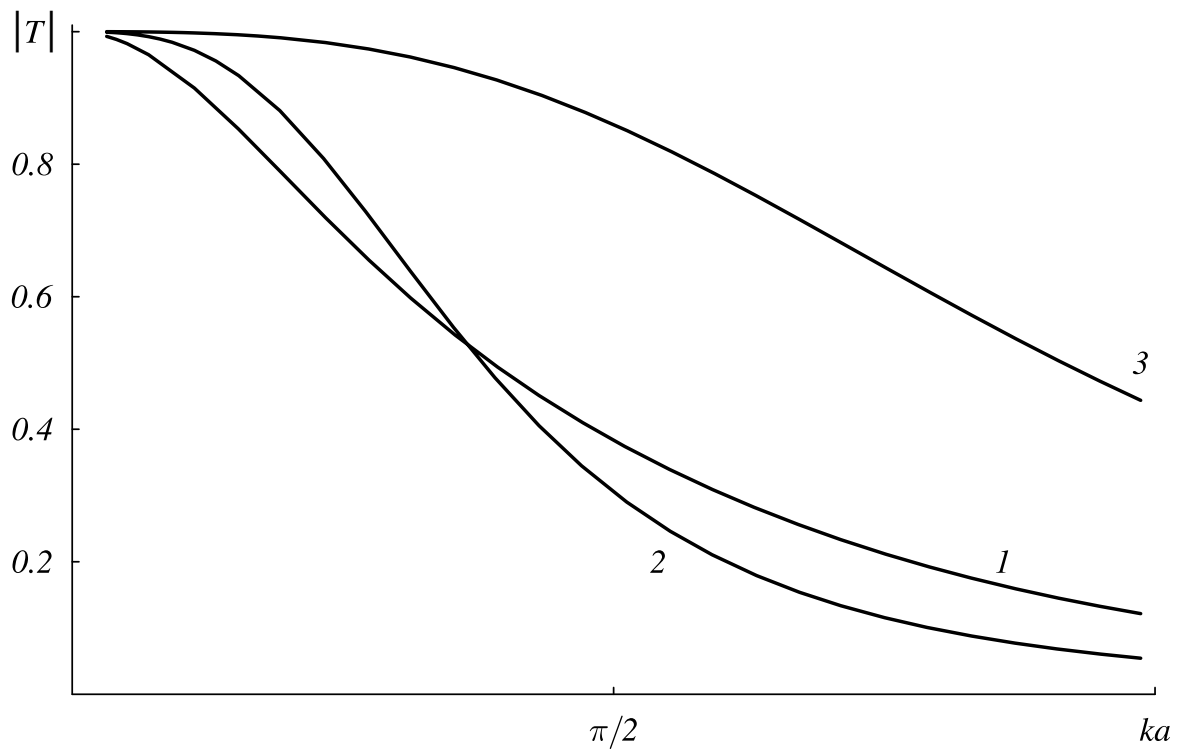

FIG. 4. Transmission coefficient vs. frequency parameter $a k<\pi$, for (relatively) large tension of membranes: $\hat{\tau} \equiv \tau /\left(\rho_{e} c^{2}\right)=10^{-2}$. Line $1: b / a=0.1 ;$ line $2: b / a=0.5 ;$ line $3: b / a=0.9$.

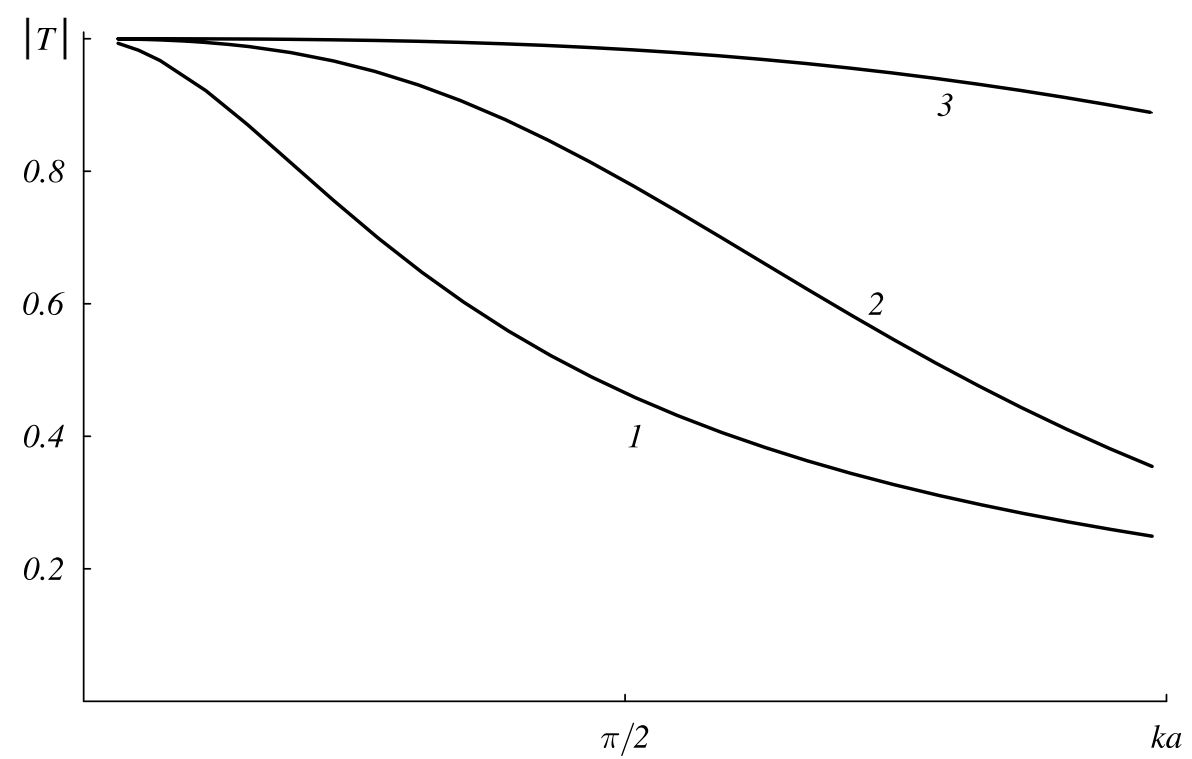

FIG. 5. Transmission coefficient vs. frequency parameter $a k<\pi$, for (relatively) very large tension of membranes: $\hat{\tau} \equiv \tau /\left(\rho_{e} c^{2}\right)=10^{-1}$. Line $1: b / a=0.1 ;$ line $2: b / a=0.5 ;$ line $3: b / a=0.9$. 
5. Wave properties of the structure. To reflect the results of our analytical approach, several examples of propagation have been considered by giving various values to the geometrical and physical parameters involved. Since we focused our attention upon dependence with respect to size and tension of the membranes, we have assumed three values for their (relative) width $b / a: b / a=0.1,0.5,0.9$; and four values for the (nondimensional) parameter $\hat{\tau} \equiv k^{2} / \Omega^{2}=\tau /\left(\rho_{e} c^{2}\right)$, which gives a (relative) measure of the tension assigned to the membrane: $\hat{\tau}=10^{-4}, 10^{-3}, 10^{-2}, 10^{-1}$. Thus, many lines for the transmission coefficient $|T|$ as a function of the frequency parameter $k a$ (in the one-mode range $(0, \pi)$ ) have been constructed operating by means of Eqs.(3.13), (4.16). For all of them, we assumed a fixed value of the ratio $\rho_{e} /\left(a \rho_{a}\right)(=10)$, meeting a typical example for the (elastic and acoustic) materials involved along with the period of the scattering structure (see Figures 2-5). Of course, in view of the approximation implied by Eqs.(3.3), the lines are more meaningful in the first half of the range. In this connection, a numerical investigation of the exact integral equation arising from Eqs.(3.2, 2.18, 2.10) has shown that the (relative) error on $|T|$ is less than $10 \%$ up to $k a \simeq 1.6$ in the (worst) case $b / a=0.9$, keeping under $5 \%$ and $2 \%$ in the remaining cases $b / a=0.5$ and $b / a=0.1$, respectively. $^{2}$ The quoted approximation can also be estimated (to a certain extent) by taking for comparison the results shown in [11], which correspond to absolutely rigid screens. Indeed, when we have now built up a line for the reflection coefficient $R(=1-T$; see Eq. $(2.11)_{1}$ ) by using a limited large value for the tension $\hat{\tau}$ (and an equivalent geometry), we practically found the same line marked with n.3 in Fig. 2 of that paper, which reflects a well-known (analytical) approach developed by Lamb and Miles; see the remarks and references therein reported.

Looking at the figures, we can observe first of all that the transmission increases with the tension given to the membrane, whatever the width and frequency of the incident wave might be. The transmission also appears to increase with respect to the membrane's width, except for the case of (relatively) small tension $\left(\hat{\tau}=10^{-4}\right.$ or $\left.10^{-3}\right)$, in which the membrane with $b / a=0.1$ allows more transmission than the membrane with $b / a=0.5$ (but less than the membrane with $b / a=0.9$ ). Moreover, as reasonably expected, with small membranes the transmission is less sensitive to the tension's values.

In any case, with a view to the possible applications in problems of noise control (see the Introduction), the more efficient attenuation of the transmission seems to occur for membranes occupying about a half of the period in the structure $(b / a=0.5)$ and preloaded with (relatively) small tension; see line 2 in Fig. 2. On the contrary, the greatest transmission is given by wide and well-stretched membranes; see line 3 in Fig. 5.

\footnotetext{
${ }^{2}$ In the second half of the range, the error grows up to $30 \%, 10 \%, 5 \%$ for $b / a=0.9,0.5,0.1$.
} 
6. Appendix. We will use the following tabular integrals (see [18]):

$$
\begin{gathered}
I_{k}=\int_{\alpha}^{\beta} \xi^{k} \sqrt{(\xi-\alpha)(\beta-\xi)} d \xi \\
=\frac{\pi^{2}(-1)^{k}(\beta-\alpha)^{2}}{(k+1)(k+2)} \sum_{\ell=0}^{k} \frac{\alpha^{\ell} \beta^{k-\ell}}{\ell !(k-\ell) ! \Gamma\left(-\frac{1}{2}-\ell\right) \Gamma\left(-\frac{1}{2}+\ell-k\right)}, \quad k=0,1,2, \ldots \\
J_{k}=\int_{\alpha}^{\beta} \xi^{k} \frac{d \xi}{\sqrt{(\xi-\alpha)(\beta-\xi)}} \\
=\pi^{2}(-1)^{k} \sum_{\ell=0}^{k} \frac{\alpha^{\ell} \beta^{k-\ell}}{\ell !(k-\ell) ! \Gamma\left(\frac{1}{2}-\ell\right) \Gamma\left(\frac{1}{2}+\ell-k\right)}, \quad k=0,1,2, \ldots \\
\int_{0}^{a} x^{\lambda-1}(a-x)^{\mu-1} \ln (a-x) d x=a^{\lambda+\mu-1} \frac{\Gamma(\lambda) \Gamma(\mu)}{\Gamma(\lambda+\mu)}[\ln a+\psi(\mu)-\psi(\lambda+\mu)] \\
\int_{0}^{a} \frac{\ln (a-c x)}{\sqrt{x(a-x)}} d x=2 \pi \ln \frac{1+\sqrt{1-c}}{2}+\pi \ln a, \\
\sqrt{\frac{a-x}{x}} \ln (d+c x) d x=\pi a \ln \frac{d+\sqrt{d(d+a c)}}{2}+\frac{\pi a}{2} \frac{\sqrt{d}-\sqrt{d+a c}}{\sqrt{d}+\sqrt{d+a c}} \\
\int_{\alpha}^{\beta} \sqrt{\frac{\xi-\alpha}{\beta-\xi}} d \xi=\frac{\pi}{2}(\beta-\alpha), \quad \int_{\alpha}^{\beta} \sqrt{\frac{\xi-\alpha}{\beta-\xi}} \frac{d \xi}{\xi}=-\pi(\sqrt{\alpha / \beta}-1) .
\end{gathered}
$$

In some of them, the (Euler's) Gamma function appears: $\Gamma(z) \equiv \int_{0}^{\infty} e^{-t} t^{z-1} d t$; useful properties of this function are

$$
\Gamma(1)=1, \quad \Gamma(z+1)=z \Gamma(z), \quad \Gamma(z) \Gamma(1-z)=\pi / \sin (\pi z) .
$$

The logarithmic derivative of $\Gamma(z)$ gives rise to the so-called psi-function:

$$
\Gamma^{\prime}(z) / \Gamma(z) \equiv \psi(z)=\psi(z+1)-1 / z ;
$$

$-\psi(1)=0.577216$ is Euler's constant. Such special functions can be evaluated by means of their well-known analytical approximations [18].

By the change $\xi=1 / \zeta$, we deduce from (A.b) that

$$
J_{-k}=J_{k-1}, \quad k=1,2, \ldots ;
$$

note also that

$$
J_{0}=\int_{\alpha}^{\beta} \frac{d \xi}{\sqrt{(\xi-\alpha)(\beta-\xi)}}=\pi=\int_{\alpha}^{\beta} \frac{d \xi}{\xi \sqrt{(\xi-\alpha)(\beta-\xi)}}=J_{-1} .
$$

The integral used in Eq.(4.10) is calculated as follows $(\xi \in L(\alpha, \beta))$ :

$$
\int_{\alpha}^{\beta} \sqrt{\frac{z-\alpha}{\beta-z}} \frac{d z}{z(\xi-z)}=\frac{1}{\xi}\left[\int_{\alpha}^{\beta} \sqrt{\frac{z-\alpha}{\beta-z}} \frac{d z}{z}+\int_{\alpha}^{\beta} \sqrt{\frac{z-\alpha}{\beta-z}} \frac{d z}{(\xi-z)}\right] ;
$$


the first integral in brackets is given by $(\text { A.f })_{2}$, while the second is

$$
\int_{\alpha}^{\beta} \sqrt{\frac{z-\alpha}{\beta-z}} \frac{d z}{(\xi-z)}=(\xi-\alpha) \int_{\alpha}^{\beta} \frac{d z}{\sqrt{(z-\alpha)(\beta-z)}(\xi-z)}-\int_{\alpha}^{\beta} \frac{d z}{\sqrt{(z-\alpha)(\beta-z)}}=-\pi,
$$

since the first integral in the right-hand side is zero $[18, n .2 .2 .6 .8]$ and the second is $J_{0}$. Thus

$$
\int_{\alpha}^{\beta} \sqrt{\frac{z-\alpha}{\beta-z}} \frac{d z}{z(\xi-z)}=-\frac{\pi}{\xi}(\sqrt{\alpha / \beta}-1)-\frac{\pi}{\xi}=-\frac{\pi}{\xi} \sqrt{\frac{\alpha}{\beta}}, \quad \xi \in L(\alpha, \beta) .
$$

Then, by using (A.d) with $a=\beta-\alpha, c=1, x=\xi-\alpha$, we have

$$
\int_{\alpha}^{\beta} \frac{\ln (\beta-\xi) d \xi}{\sqrt{(\xi-\alpha)(\beta-\xi)}}=\int_{0}^{\beta-\alpha} \frac{\ln (\beta-\alpha-x)}{\sqrt{x(\beta-\alpha-x)}} d x=\pi \ln \frac{\beta-\alpha}{4} .
$$

By the same reasoning, but with $c=(\alpha-\beta) / \alpha$, it follows that

$$
\begin{aligned}
& \int_{\alpha}^{\beta} \frac{\ln \xi d \xi}{\sqrt{(\xi-\alpha)(\beta-\xi)}}=\int_{0}^{\beta-\alpha} \frac{\ln (\alpha+x)}{\sqrt{x(\beta-\alpha-x)}} d x \\
& \quad=\int_{0}^{\beta-\alpha} \frac{\ln [\alpha /(\beta-\alpha)]+\ln [\beta-\alpha-x(\alpha-\beta) / \alpha]}{\sqrt{x(\beta-\alpha-x)}} d x \\
& \quad=\pi \ln \frac{\alpha}{\beta-\alpha}+2 \pi \ln \frac{1+\sqrt{1-(\alpha-\beta) / \alpha}}{2}+\pi \ln (\beta-\alpha)=2 \pi \ln \frac{\sqrt{\alpha}+\sqrt{\beta}}{2} .
\end{aligned}
$$

Putting $\xi=1 / \zeta$ and recalling: (A.6) with $\beta-\xi=\zeta-\alpha,(\mathrm{A} .2),(\mathrm{A} .7)$, we get

$$
\begin{gathered}
\int_{\alpha}^{\beta} \frac{\ln (\beta-\xi) d \xi}{\xi \sqrt{(\xi-\alpha)(\beta-\xi)}}=\int_{\alpha}^{\beta} \frac{\ln (\zeta-\alpha)-\ln \alpha-\ln \zeta}{\sqrt{(\zeta-\alpha)(\beta-\zeta)}} d \zeta \\
=\pi \ln \frac{\beta-\alpha}{4}-\pi \ln \alpha-2 \pi \ln \frac{\sqrt{\alpha}+\sqrt{\beta}}{2}=\pi\left[\ln \frac{\beta-\alpha}{\alpha}-2 \ln (\sqrt{\alpha}+\sqrt{\beta})\right]
\end{gathered}
$$

moreover, putting $\xi=1 / \zeta$ and recalling (A.7):

$$
\int_{\alpha}^{\beta} \frac{\ln \xi d \xi}{\xi \sqrt{(\xi-\alpha)(\beta-\xi)}}=-\int_{\alpha}^{\beta} \frac{\ln \zeta d \zeta}{\sqrt{(\zeta-\alpha)(\beta-\zeta)}}=-2 \pi \ln \frac{\sqrt{\alpha}+\sqrt{\beta}}{2} .
$$

Then, we need to calculate the integrals $N_{k}(\xi), k=0,1,2, \ldots$ (see Eqs.(4.15)). Let us begin with $N_{0}(\xi)$; it follows that

$$
\begin{aligned}
N_{0}(\xi) & =\int_{\alpha}^{\beta} \frac{\sqrt{(\tau-\alpha)(\beta-\tau)}}{\xi-\tau} d \tau=(\beta-\xi) \int_{\alpha}^{\beta} \sqrt{\frac{\tau-\alpha}{\beta-\tau}} \frac{d \tau}{\xi-\tau}+\int_{\alpha}^{\beta} \sqrt{\frac{\tau-\alpha}{\beta-\tau}} d \tau \\
& =(\beta-\xi)(-\pi)+\frac{\pi}{2}(\beta-\alpha)=\pi\left(\xi-\cos \frac{\pi d}{a}\right),
\end{aligned}
$$


thanks to the integrals (A.4), (A.f $)_{1}$. For $k=1,2, \ldots$, writing

$$
\frac{\tau^{k}}{\xi-\tau}=\frac{\xi^{k}}{\xi-\tau}-\sum_{j=0}^{k-1} \xi^{k-j-1} \tau^{j}
$$

we have

$$
N_{k}(\xi)=\xi^{k} N_{0}(\xi)-\sum_{j=0}^{k-1} \xi^{k-j-1} I_{j}, \quad k=1,2, \ldots
$$

Let us pass to the integrals in Eq.(4.17), for $k=1,2, \ldots$ (for $k=0$ see (A.6,A.7)); writing

$$
\xi^{k}=\sum_{j=0}^{k}\left(\begin{array}{l}
k \\
j
\end{array}\right)(\xi-\alpha)^{k-j} \alpha^{j}
$$

and using (A.c) with $a=\beta-\alpha, x=\xi-\alpha, \lambda=1 / 2+k-j, \mu=1 / 2$, we get

$$
\begin{aligned}
P_{k} & =\sum_{j=0}^{k}\left(\begin{array}{c}
k \\
j
\end{array}\right) \alpha^{j} \int_{\alpha}^{\beta} \ln (\beta-\xi)(\xi-\alpha)^{k-j-1 / 2}(\beta-\xi)^{-1 / 2} d \xi=\sum_{j=0}^{k}\left(\begin{array}{c}
k \\
j
\end{array}\right) \alpha^{j}(\beta-\alpha)^{k-j} \\
& \times\left\{\frac{\Gamma(1 / 2+k-j) \Gamma(1 / 2)}{\Gamma(1+k-j)}[\ln (\beta-\alpha)+\psi(1 / 2)-\psi(1+k-j)]\right\} .
\end{aligned}
$$

To calculate $Q_{k}$, let us introduce the following notation:

$T_{k}=\int_{\alpha}^{\beta} \xi^{k} \sqrt{\frac{\beta-\xi}{\xi-\alpha}} \ln \xi d \xi, R_{k}=\int_{\alpha}^{\beta} \xi^{k} \sqrt{\frac{\xi-\alpha}{\beta-\xi}} \ln \xi d \xi, S_{k}=\int_{\alpha}^{\beta} \xi^{k} \sqrt{(\xi-\alpha)(\beta-\xi)} \ln \xi d \xi$.

In particular, putting $\xi=x+\alpha$, it follows that

$$
\begin{aligned}
T_{0} & =\int_{\alpha}^{\beta} \sqrt{\frac{\beta-\xi}{\xi-\alpha}} \ln \xi d \xi=\int_{0}^{\beta-\alpha} \frac{(\beta-\alpha-x)}{\sqrt{x}} \ln (\alpha+x) d x \\
& =\pi\left[(\beta-\alpha) \ln \frac{\sqrt{\alpha}+\sqrt{\beta}}{2}+1-\frac{\alpha+\beta}{2}\right],
\end{aligned}
$$

thanks to (A.e) with $a=\beta-\alpha, d=\alpha, c=1$. We easily get

$$
2 Q_{k}=(\alpha+\beta) Q_{k-1}+\left(R_{k-1}-T_{k-1}\right) ;
$$

moreover, by suitable integration by parts in $T_{k}$, we can deduce that

$$
T_{k}=-2 k S_{k-1}-2 I_{k-1}+R_{k}
$$

where integrals of type (A.a) appear. It follows that

$$
S_{k-2}=\int_{\alpha}^{\beta} \xi^{k-2} \frac{(\xi-\alpha)(\beta-\xi)}{\sqrt{(\xi-\alpha)(\beta-\xi)}} \ln \xi d \xi=-Q_{k}+(\alpha+\beta) Q_{k-1}-Q_{k-2},
$$

so that Eqs.(A.15, A.16, A.17) finally give an iteration formula for $Q_{k}$ from $k \geq 2$ :

$$
2 k Q_{k}=(2 k-1)(\alpha+\beta) Q_{k-1}-(2 k-2) Q_{k-2}+2 I_{k-2} .
$$


The starting values are (see (A.7, A.14)):

$$
Q_{0}=2 \pi \ln \frac{\sqrt{\alpha}+\sqrt{\beta}}{2}, \quad Q_{1}=-T_{0}+\beta Q_{0} .
$$

Continuing on, we have (putting $\xi=1 / \zeta$ and then $\zeta-\alpha=\beta-\eta$ ) :

$$
\begin{aligned}
P_{-k} & =\int_{\alpha}^{\beta} \zeta^{k-1} \frac{\ln (\zeta-\alpha)-\ln \alpha-\ln \zeta}{\sqrt{(\zeta-\alpha)(\beta-\zeta)}} d \zeta \\
& =\int_{\alpha}^{\beta}[(\alpha+\beta)-\eta]^{k-1} \frac{\ln (\beta-\eta)}{\sqrt{(\eta-\alpha)(\beta-\eta)}} d \eta-(\ln \alpha) J_{k-1}-Q_{k-1} .
\end{aligned}
$$

The first integral on the right-hand side is calculated by expanding the term in the power $k-1$, so that we easily get:

$$
P_{-k}=\sum_{j=0}^{k-1}\left(\begin{array}{c}
k-1 \\
j
\end{array}\right)(\alpha+\beta)^{k-1-j}(-1)^{j} P_{j}-(\ln \alpha) J_{k-1}-Q_{k-1}, k=1,2, \ldots
$$

Moreover, it follows that (putting $\xi=1 / \zeta)$ :

$$
Q_{-k}=-Q_{k-1}, \quad k=1,2, \ldots
$$

Acknowledgments. This paper has been supported by "Gruppo Nazionale per la Fisica Matematica" of the Italian "INdAM", and, in part, by the "Russian Foundation for Basic Researches" (Grant 05-01-00155). The authors are grateful to the reviewer for useful criticism.

\section{REFERENCES}

[1] R.L. Bisplinghoff, H. Ashley, R.L. Halfman, "Aeroelasticity", Addison-Wesley, Cambridge (Massachusetts), 1955.

[2] A.H. Flax, Aero and hydro-elasticity, in: "Structural Mechanics". Proc. 1st Symp. Naval Struct. Mech. (ed. J.N. Goodier, N.J. Hoff), Pergamon Press, Oxford, 1960. MR0112384 (22:3235)

[3] H.H. Bleich, Dynamics interaction between structures and fluids, in: "Structural Mechanics". Proc. 1st Symp. Naval Struct. Mech. (ed. J.N. Goodier, N.J. Hoff), Pergamon Press, Oxford, 1960. MR0112381 (22:3232)

[4] C.M. Harris, "Handbook of Acoustical Measurements and Noise Control" (3rd ed.), Acoust. Soc. America, Woodbury (NY), 1998.

[5] A.D. Pierce, "Acoustics", Acoust. Soc. America, Woodbury (NY), 1998.

[6] M.C. Junger, Acoustic fluid-elastic structure interactions: basic concepts, Computers and Structures 65 (1997) 287-293.

[7] M.C. Junger, D. Feit, "Sound, Structures and their Interaction", MIT Press, Massachusetts, 1986.

[8] S. Kukathasan, S. Pellegrino, Vibration of prestressed membrane structures in air, A IA A 2002$1368,1-11$.

[9] "Aeroacoustics of Flight Vehicles", vol. 2 (ed. H.H. Hubbard), Acoust. Soc. America, Woodbury (NY), 1995.

[10] L. Cremer, H.A. Müller, "Principles and Applications of Room Acoustics", vols. 1, 2, Applied Science, London, 1982.

[11] E. Scarpetta, M.A. Sumbatyan, Explicit analytical results for one-mode normal reflection and transmission by a periodic array of screens, J. Math. Anal. Appl. 195 (1995) 736-749. MR1356640 (96h:76074) 
[12] E. Scarpetta, M.A. Sumbatyan, Explicit analytical results for one-mode oblique penetration into a periodic array of screens, IMA J. Appl. Math. 56 (1996) 109-120. MR1401176 (97e:76081)

[13] F.G. Leppington, Acoustic scattering by membranes and plates with line constraints, J. Sound Vibr. 58 (1978) 319-332.

[14] I.D. Abrahams, Scattering of sound by an elastic plate with flow, J. Sound Vibr. 89 (1983) 213-231. MR720640 (84j:76039)

[15] I.D. Abrahams, Diffraction by a semi-infinite membrane in the presence of a vertical barrier, $J$. Sound Vibr. 111 (1986) 191-207.

[16] P.D. Metcalfe, Ribbed elastic structures under a mean flow, Proc. R. Soc. A 461 (2005) 913-931. MR2144610 (2005k:74029)

[17] F.D. Gakhov, "Boundary Value Problems", Pergamon Press, Oxford, 1966. MR0198152 (33:6311)

[18] A.P. Prudnikov, Y.A. Brychkov, O.I. Marichev. "Integrals and Series" vol. 2, Gordon and Breach Science Publishers, Amsterdam, 1986. MR874987 (88f:00014) 